\title{
Height correction in the measurement of solar differential rotation determined by coronal bright points
}

\author{
R. Brajša ${ }^{1,2, \star}$, H. Wöhl ${ }^{1}$, B. Vršnak ${ }^{2}$, V. Ruždjak ${ }^{2}$, F. Clette ${ }^{3}$, J.-F. Hochedez ${ }^{3}$, and D. Roša ${ }^{4}$ \\ ${ }^{1}$ Kiepenheuer-Institut für Sonnenphysik (KIS), Schöneckstr. 6, 79104 Freiburg, Germany \\ e-mail: rbrajsa@kis.uni-freiburg.de; hw@kis.uni-freiburg.de \\ ${ }^{2}$ Hvar Observatory, Faculty of Geodesy, University of Zagreb, Kačićeva 26, 10000 Zagreb, Croatia \\ e-mail: romanb@geof.hr; bvrsnak@geof.hr; vruzdjak@geof.hr \\ 3 Observatoire Royal de Belgique (ORB), Ave. Circulaire 3, 1180 Bruxelles, Belgium \\ e-mail: Frederic.clette@oma.be; hochedez@oma.be \\ ${ }^{4}$ Zagreb Astronomical Observatory, Opatička 22, 10000 Zagreb, Croatia \\ e-mail: drosa@hpd.botanic.hr
}

Received 16 July 2003 / Accepted 3 October 2003

\begin{abstract}
Full-disc solar images obtained with the Extreme Ultraviolet Imaging Telescope (EIT) on board the Solar and Heliospheric Observatory (SOHO) are used to analyse solar differential rotation by tracing coronal bright points for the period June 4, 1998 to May 22, 1999. A method for the simultaneous determination of the true solar synodic rotation velocity and the height of the tracers is applied to data sets analysed with interactive and automatic methods. The calculated height of coronal bright points is on average 8000-12000 km above the photosphere. Corrected rotation velocities are transformed into sidereal ones and compared with results from the literature, obtained with various methods and tracers. The differential rotation profile determined by coronal bright points with the interactive method corresponds roughly to the profile obtained by correlating photospheric magnetic fields and the profile obtained from the automatic method corresponds roughly to the rotation of sunspot groups. This result is interpreted in terms of the differences obtained in the latitudinal distribution of coronal bright points using the two methods.
\end{abstract}

Key words. Sun: corona - Sun: UV radiation - Sun: rotation

\section{Introduction}

Observational characteristics and physical properties of coronal bright points in various wavelength ranges were recently summarized by Brown et al. (2001), Longcope et al. (2001) and Madjarska et al. (2003). Note that coronal bright points are magnetic tracers associated with bipolar magnetic features (Webb et al. 1993); 1/3 of them lie over ephemeral regions (new emerging regions of magnetic flux) and the remaining $2 / 3$ lie above cancelling magnetic features (consisting of opposite polarity fragments that approach and disappear). A converging flux model of an X-ray bright point connected to an associated cancelling magnetic structure was developed by Priest et al. (1994). Coronal bright points were also considered to be indirect signatures of the solar dynamo located at the bottom of the convective zone (Golub et al. 1981; Gilman \& DeLuca 1986). Furthermore, their significance for the coronal heating was also discussed (Gokhale 1975; Priest et al. 1994; Longcope et al. 2001).

Send offprint requests to: R. Brajša,

e-mail: rbrajsa@kis.uni-freiburg.de

* Alexander von Humboldt Research Fellow at KIS.
In the determination of the solar rotation it is important to take into account the height of the tracers. The value of the necessary height correction increases with the distance from the solar disc centre (Roša et al. 1998) and the measured rotation velocity of coronal tracers cannot be reliably compared with the velocities obtained using other tracers and methods if the height correction is not taken into account properly. This is especially important at higher latitudes, where in addition to false velocity determination the apparent (measured) and true latitude also significantly differ for tracers lying above the solar photosphere (Brajša et al. 1991, 1997; Roša et al. 1998). The height correction was performed in different ways for $\mathrm{H} \alpha$ filaments (d'Azambuja \& d'Azambuja 1948; Adams \& Tang 1977; Förster 1979; Brajša et al. 1991; Vršnak et al. 1999) and for radio and microwave tracers (Liu \& Kundu 1976; Aschwanden \& Bastian 1994a, 1994b; Aschwanden et al. 1995; Brajša et al. 1999, 2000a). On the other hand, the corrections due to the Wilson depression of sunspots are relatively small, but cannot be neglected close to the solar limb (Balthasar \& Wöhl 1983).

In this paper we continue our investigation of solar differential rotation determined by tracing coronal bright points in SOHO-EIT images during the 1998/99 observing period. 
The interactive and the automatic methods of data reduction and the application of the improved solar disc coordinates (Auchère et al. 1998) were introduced and described in Brajša et al. (2001a). In Brajša et al. (2002a) the results on differential rotation for the two methods, for the northern and southern solar hemispheres, and for the three subtypes of tracers (pointlike structures, small loops, and small active regions) were presented and compared. Properties of the solar velocity field indicated by motions of coronal bright points expressed through the rotation velocity residual, meridional motions and Reynolds stresses were discussed in Vršnak et al. (2003).

We apply a method to determine simultaneously the true solar rotation rate and the height of tracers (Roša et al. 1998) to our set of solar rotation measurements tracing coronal bright points. By this method it is possible to calculate the average height of all tracers in different latitude bands. The interactive method of data reduction also provides the possibility to distinguish between the various tracer subtypes (point-like structures, small loops, and small active regions) which do not on average necessarily have the same height and, consequently, the same value of the height correction. On the other hand, various automatic procedures of data reduction (e.g., Brajša et al. 2001a; Wöhl et al. 2001; Gissot et al. 2003) are very useful because of the large SOHO-EIT data set (Hochedez et al. 2001). In such cases it is important to know statistical properties of the tracers including their height. This will be especially important when the appropriate automatic method will be applied to the data from the whole SOHO lifetime. It is important to extend the solar rotational analysis to higher latitudes (e.g., Fisk 1996) and this is one of the primary goals of our study. This information can be provided by study of coronal bright points, since they are widely distributed over solar latitudes.

Some preliminary results, obtained using a small subset of our data without improved solar disc coordinates and not taking into account the latitudinal dependence of the correction, have been described by Brajša et al. (2000b). In that paper we found that coronal bright points had no significant height above the solar radius used in the coordinate transformation ( $R=704000 \mathrm{~km}$ ), which is $8000 \mathrm{~km}$ larger than the radius of the solar photosphere. In the present paper we check the reliability of that result using a larger data set and applying the analytical method consistently.

\section{The data set and reduction methods}

The data set consists of 463 full-disc solar filtergrams recorded in the $\mathrm{Fe} X \mathrm{XV}$ line at the wavelength of $28.4 \mathrm{~nm}$ with the EIT instrument on board the SOHO spacecraft during June, November, and December 1998 and March, April, and May 1999. The images were taken with a regular cadence of 6 hours. The data were reduced using two methods, the interactive and the automatic one.

Using the interactive method, coronal bright points are visually traced in consecutive images on a computer screen. This was carried out by several observers. Some of them performed the tracing in shorter time intervals only, while others followed the bright points as long as possible. So, for the whole observing period, June 4, 1998 to May 22, 1999, two data sets were established with the interactive method. For the "data set 1" the tracing was performed in up to 11 consecutive images and for the "data set 2 " the tracing was performed in up to 24 consecutive images. In both cases the rotation velocities were calculated by the linear least-squares fit of the central meridian distances, $\lambda$ (also denoted as CMD), as a function of time, $\lambda(t)$, and at least three images were used to determine the velocity of each bright point. For each velocity value the mean $\lambda$ during the tracing was calculated. Since the height correction depends on CMD and it is important to measure as many rotation velocities for different CMDs in each latitude band as possible, the more abundant data set 1 will be used in the present paper. In the case of the interactive method (visual tracing) it is also possible to identify the tracer subtype: point-like structures, small loops, and small active regions.

The automatic method of data reduction is based on the Interactive Data Language (IDL) procedure "Regions Of Interest (ROI) segmentation". The ROI procedure was applied to triplets of images taken every 6 hours. The numerical ROI parameters of the automatic method (sharpness of the subimages, their circumference range and the intensity range of their brightness) were chosen to resemble the interactive method as much as possible. The ROI parameters that were used in the actual analysis were given by Brajša et al. (2002a). We point out that it is not possible to identify different tracer subtypes applying the automatic method.

Further technical details about data selection and preparation of images for the reduction were given in Brajša et al. (2001a; 2002a).

\section{Height correction}

\subsection{The method}

We now describe briefly the method for the simultaneous determination of the corrected solar rotation velocity and the height of tracers according to Roša et al. (1998). Quantities denoted with an asterisk $\left(^{*}\right)$ are the measured ones and without an asterisk the corrected ones.

We introduce first the parameter $\beta$, which connects the height parameter $\epsilon=h / R$, where $h$ is the height above the solar surface and $R$ the solar radius, with the observed latitude, $\psi^{*}$, and corrected (true) latitude of the tracer, $\psi$

$\beta=(1+\epsilon) \frac{\cos \psi}{\cos \psi^{*}}$.

This is Eq. (17b) in Roša et al. (1998), which can for the case $B_{0} \approx 0$, where $B_{0}$ is the heliographic latitude of the solar disc centre, be written as

$\beta=\frac{\sqrt{(1+\epsilon)^{2}-\sin ^{2} \psi^{*}}}{\cos \psi^{*}}=$ constant.

This is Eq. (20b) in Roša et al. (1998). The relative deviation of the parameter $\beta$ from a constant value due to the change of the projected heliographic latitude is less than $2 \%$ even in the most inconvenient cases when $B_{0}$ reaches the maximum value and the tracer is close to the limb (Roša et al. 1998). 
Now we connect the parameter $\beta$ with the mean observed central meridian distance $\lambda^{*}$, and corresponding observed rotation velocity $\omega^{*}$, and corrected rotation velocity $\omega$, of the measured tracer

$\omega_{i}^{*}=\frac{\sqrt{\beta^{2}-\sin ^{2} \lambda_{i}^{*}}}{\cos \lambda_{i}^{*}} \omega$.

This is Eq. (21d) in Roša et al. (1998).

Further, the corrected rotation velocity $\omega$ and the parameter $\beta$ can be calculated by

$\omega^{2}=\frac{a b-c d}{e-d^{2}}$,

$\beta^{2}=\frac{b-d+c / \omega^{2}}{b}$,

which are Eqs. (33a) and (33b) in Roša et al. (1998), respectively. The following abbreviations were introduced

$a=\sum_{i=1}^{N} \omega_{i}^{* 2}$

$b=\sum_{i=1}^{N} \frac{1}{\cos ^{4} \lambda_{i}^{*}}$,

$c=\sum_{i=1}^{N} \frac{\omega_{i}^{* 2}}{\cos ^{2} \lambda_{i}^{*}}$,

$d=\sum_{i=1}^{N} \frac{1}{\cos ^{2} \lambda_{i}^{*}}$,

$e=\sum_{i=1}^{N} \frac{N}{\cos ^{4} \lambda_{i}^{*}}$,

where the summation refers to 10 -deg latitude bins and $N$ is the number of measured pairs of velocities $\omega_{i}^{*}$ and mean central meridian distances $\lambda_{i}^{*}$, in each bin.

Finally, the true height of the tracer is calculated by

$h=R\left(\sqrt{\beta^{2} \cos ^{2} \psi^{*}+\sin ^{2} \psi^{*}}-1\right)$,

where $\psi^{*}$ is the mean latitude value for each latitude bin

$\psi^{*}=\frac{1}{N} \sum_{i=1}^{N} \psi_{i}^{*}$

These two expressions are Eqs. (34) and (35) from Roša et al. (1998). Equation (11), as well as Eq. (2), hold in the approximation $B_{0} \approx 0$. The mean value of the measured rotation velocities is calculated by

$\omega^{*}=\frac{1}{N} \sum_{i=1}^{N} \omega_{i}^{*}$,

for each latitude bin. The corrected latitude $\psi$ is calculated by

$\cos \psi=\frac{\beta \cos \psi^{*}}{\sqrt{\beta^{2} \cos ^{2} \psi^{*}+\sin ^{2} \psi^{*}}}$

This formula is obtained by combining Eqs. (1) and (2).
Table 1. Effective heights of coronal bright points calculated for various latitude bins using Eqs. (11) and (12) for the interactive method. The results for all data and for the subsample of point-like structures (PLS) are presented.

\begin{tabular}{|c|c|c|}
\hline Latitude $\psi(\operatorname{deg})$ & $\begin{array}{r}\text { all subtypes } \\
\text { height } h(\mathrm{~km})\end{array}$ & $\begin{array}{r}\text { PLS only } \\
\text { height } h(\mathrm{~km})\end{array}$ \\
\hline $0.0 \leq \psi<10.0$ & 3997 & 4911 \\
\hline $10.0 \leq \psi<20.0$ & -184 & -361 \\
\hline $20.0 \leq \psi<30.0$ & 3607 & 5821 \\
\hline $30.0 \leq \psi<40.0$ & 1842 & 3284 \\
\hline $40.0 \leq \psi<50.0$ & 9332 & 16758 \\
\hline $50.0 \leq \psi<60.0$ & 3396 & 2819 \\
\hline $60.0 \leq \psi<70.0$ & 8868 & 9368 \\
\hline
\end{tabular}

\subsection{The calculation procedure}

The calculation of the height correction proceeds in the following way. All measured rotation velocities (without any velocity filter, Brajša et al. 2002a) with the corresponding mean values of the CMD are divided in 10-deg latitude bins. Now, for each latitude bin the mean value of the corrected rotation velocity $\omega$ and the parameter $\beta$ are calculated according to Eqs. (4) and (5), respectively. The measured rotation velocities $\omega_{i}^{*}$ are now fitted as a function of the measured central meridian distance $\lambda_{i}^{*}$ using Eq. (3) where the corrected (true) rotation velocity $\omega$ and the value of the parameter $\beta$ are taken as fit parameters in each latitude bin. All measurements with the corresponding curves fitted in this way are presented separately in Figs. 1 and 2, for the interactive and automatic methods, respectively. The scatter of data points in Figs. 1 and 2 provides some insight into the errors of measurements, indicating also the statistical nature of the height correction procedure.

Further, the height of the tracer above the solar surface (expressed in units of the solar radius $R$ ) is calculated according to Eq. (11) for each latitude bin (in Table 1 the results for the interactive method are presented as an example) taking into account the mean latitude of all measurements in each bin using Eq. (12). Finally, the corrected mean latitude for each bin is calculated using Eq. (14).

For each latitude bin we now have the mean observed values of the latitude and the rotation velocity, $\psi^{*}$ and $\omega^{*}$, calculated using Eqs. (12) and (13), and the corresponding corrected values, $\psi$ and $\omega$, calculated using Eqs. (4) and (14). From these values the corrections specific for each latitude bin are calculated as $\Delta \omega=\omega-\omega^{*}$ and $\Delta \psi=\psi-\psi^{*}$ and used to correct all individual measured pairs of the rotation velocity and latitude. In this way the corrected data sets for each method of data reduction, the interactive and the automatic one, were established.

\section{Results}

\subsection{Differential rotation}

The raw and corrected data for both methods of data reduction are further treated in the usual way, applying the two-step 

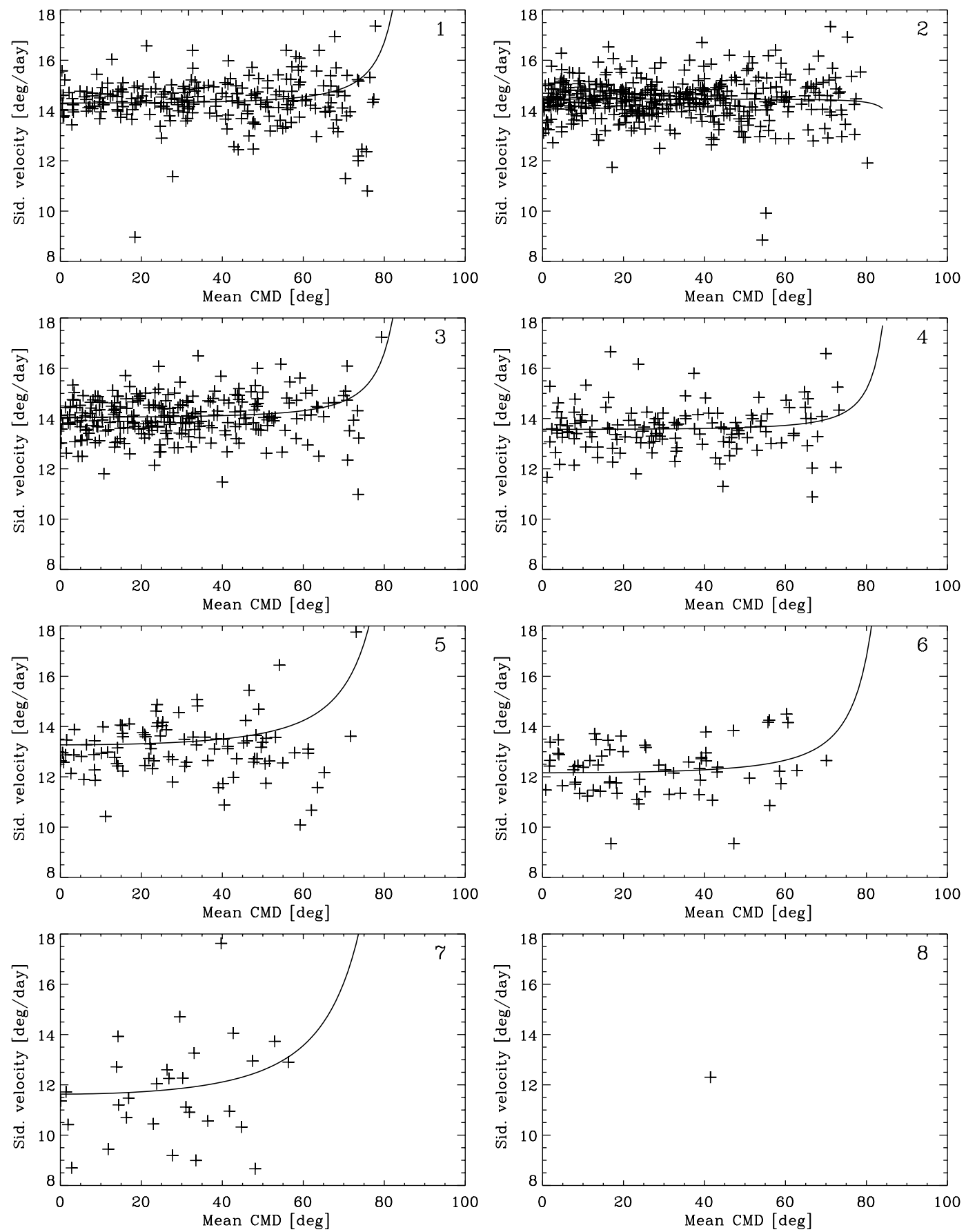

Fig. 1. Observed solar sidereal rotation velocities as a function of the CMD for 10-deg latitude bins: panel 1 for the latitudes $0.0 \leq \psi<10.0$ deg, panel 2 for the latitudes $10.0 \leq \psi<20.0 \mathrm{deg}$, etc. Results obtained with the interactive method are presented for both solar hemispheres treated together. For details see text.

velocity filter (Brajša et al. 2002a) and calculating the parameters of the solar differential rotation, $A, B$, and $C$ according to the standard formula

$\omega(\psi)=A+B \sin ^{2} \psi+C \sin ^{4} \psi$,

where $\omega$ is the sidereal rotation velocity in deg/day and $\psi$ the heliographic latitude in degrees.

The parameters are presented in Tables 2-5 for the interactive and the automatic method and for the raw and corrected data sets. The cases with only the first two parameters $(C=0)$ from Eq. (15), with all three parameters $(B \neq C)$ and imposing $B=C$ are calculated. In the last of the above-mentioned cases, Eq. (15) was transformed to $\omega(\psi)=y(x)=A+B\left(x+x^{2}\right)$ with the substitution $x=\sin ^{2} \psi$. Now we take $x+x^{2}=x^{\prime}$ leading to $y\left(x^{\prime}\right)=A+B x^{\prime}$, which is now fitted with the linear least-square method, as usual (e.g., Brajša et al. 1997).

The differential rotation curves for the interactive and automatic methods are shown in Figs. 3 and 4, respectively. For both cases data sets with and without the height correction are presented. 

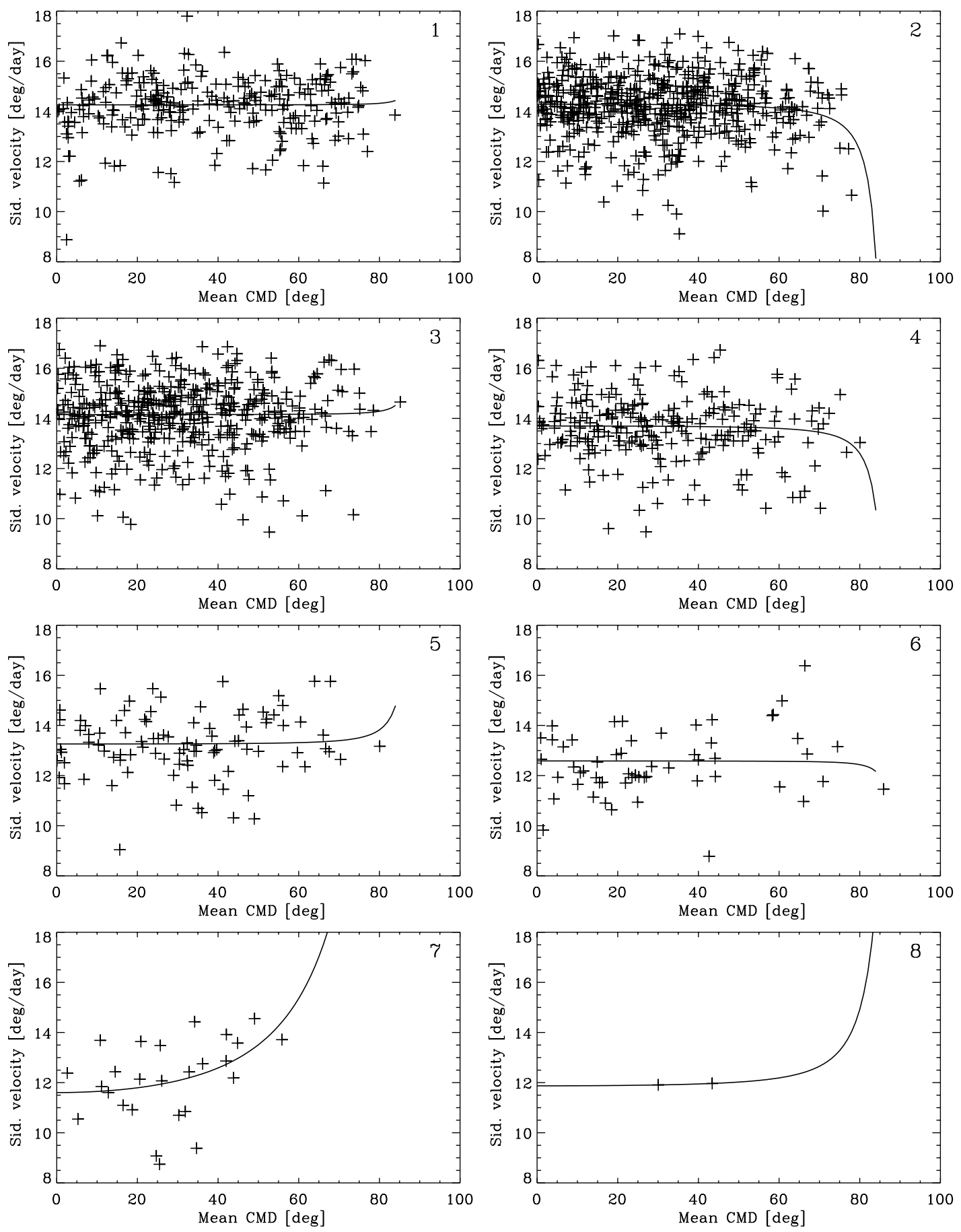

Fig. 2. As Fig. 1 for the automatic method.

\subsection{Height of coronal bright points}

The effective heights of coronal bright points are calculated for each 10-deg latitude bin using Eq. (11). The first seven latitude bins, i.e., latitudes up to $70 \mathrm{deg}$ are taken into account and the mean values with standard errors (expressed as $\sigma / \sqrt{N}$, where $\sigma$ is the standard deviation and $N$ the number of data points in the bin) are calculated.

The calculated heights refer to the solar radius $R=$ $701500 \mathrm{~km}$ used in the coordinate transformation. This effective solar radius was obtained in the following way. From the

solar radius in pixels, the solar radius in arcsec is calculated taking into account that 1 pixel corresponds to 2.629 arcsec. Then the distance from the spacecraft to the Sun was calculated from the coordinates of the SOHO spacecraft in the solar system, and used to convert the solar radius in arcsec to the one in kilometers.

We obtain following heights relative to the mentioned solar radius $R=701500 \mathrm{~km}$ : for the interactive method $\bar{h}=4400 \mathrm{~km} \pm 1300 \mathrm{~km}$ and for the automatic method $\bar{h}=$ $2800 \mathrm{~km} \pm 3300 \mathrm{~km}$. In the interactive method it is also possible to distinguish between various tracer subtypes, point-like 
Table 2. Differential rotation parameters $A, B$, and $C$ from Eq. (15) for the interactive method, without the height correction. The sidereal parameters and their standard errors $(M)$ are expressed in deg/day for three cases (see remarks). Both solar hemispheres are treated together and the number of tracers $n=1179$ (after the velocity filter was applied).

\begin{tabular}{llll}
\hline \hline$A \pm M_{A}$ & $-B \pm M_{B}$ & $-C \pm M_{C}$ & Remark \\
\hline $14.591 \pm 0.028$ & $3.18 \pm 0.10$ & 0 & $C=0$ \\
$14.549 \pm 0.036$ & $2.59 \pm 0.33$ & $0.89 \pm 0.47$ & $B \neq C$ \\
$14.495 \pm 0.026$ & $1.89 \pm 0.06$ & $1.89 \pm 0.06$ & $B=C$ \\
\hline
\end{tabular}

Table 3. As Table 2 with the height correction (interactive method). $n=1182$.

\begin{tabular}{llll}
\hline \hline$A \pm M_{A}$ & $-B \pm M_{B}$ & $-C \pm M_{C}$ & Remark \\
\hline $14.561 \pm 0.029$ & $3.68 \pm 0.11$ & 0 & $C=0$ \\
$14.496 \pm 0.037$ & $2.77 \pm 0.34$ & $1.40 \pm 0.50$ & $B \neq C$ \\
$14.454 \pm 0.027$ & $2.22 \pm 0.07$ & $2.22 \pm 0.07$ & $B=C$ \\
\hline
\end{tabular}

Table 4. As Table 2 for the automatic method (without the height correction). $n=1500$.

\begin{tabular}{llll}
\hline \hline$A \pm M_{A}$ & $-B \pm M_{B}$ & $-C \pm M_{C}$ & Remark \\
\hline $14.530 \pm 0.032$ & $2.68 \pm 0.13$ & 0 & $C=0$ \\
$14.460 \pm 0.042$ & $1.79 \pm 0.36$ & $1.38 \pm 0.52$ & $B \neq C$ \\
$14.446 \pm 0.029$ & $1.63 \pm 0.08$ & $1.63 \pm 0.08$ & $B=C$ \\
\hline
\end{tabular}

Table 5. As Table 3 for the automatic method (with the height correction). $n=1495$.

\begin{tabular}{llll}
\hline \hline$A \pm M_{A}$ & $-B \pm M_{B}$ & $-C \pm M_{C}$ & Remark \\
\hline $14.677 \pm 0.033$ & $3.10 \pm 0.14$ & 0 & $C=0$ \\
$14.549 \pm 0.043$ & $1.43 \pm 0.38$ & $2.75 \pm 0.58$ & $B \neq C$ \\
$14.591 \pm 0.030$ & $1.94 \pm 0.08$ & $1.94 \pm 0.08$ & $B=C$ \\
\hline
\end{tabular}

structures, small loops and small active regions. The mean height of the point-like structures is $\bar{h}=6100 \mathrm{~km} \pm 2100 \mathrm{~km}$, while the subsets of the other two tracers are too small to get reliable results. We note that the above-mentioned heights are rounded up to $100 \mathrm{~km}$ (see also Table 1).

Finally, the transformed heights of coronal bright points above the solar photosphere, having the radius of $696000 \mathrm{~km}$, are approximately $9900 \mathrm{~km}$ for the interactive method, $8300 \mathrm{~km}$ for the automatic method and $11600 \mathrm{~km}$ for the pointlike tracer subtype.

\section{Comparison with other data, discussion, and conclusions}

In Table 6 representative solar differential rotation parameters from the present work are compared to selected results from the literature, where the solar rotation was determined tracing sunspots, $\mathrm{H} \alpha$ filaments, coronal bright points, microwave low

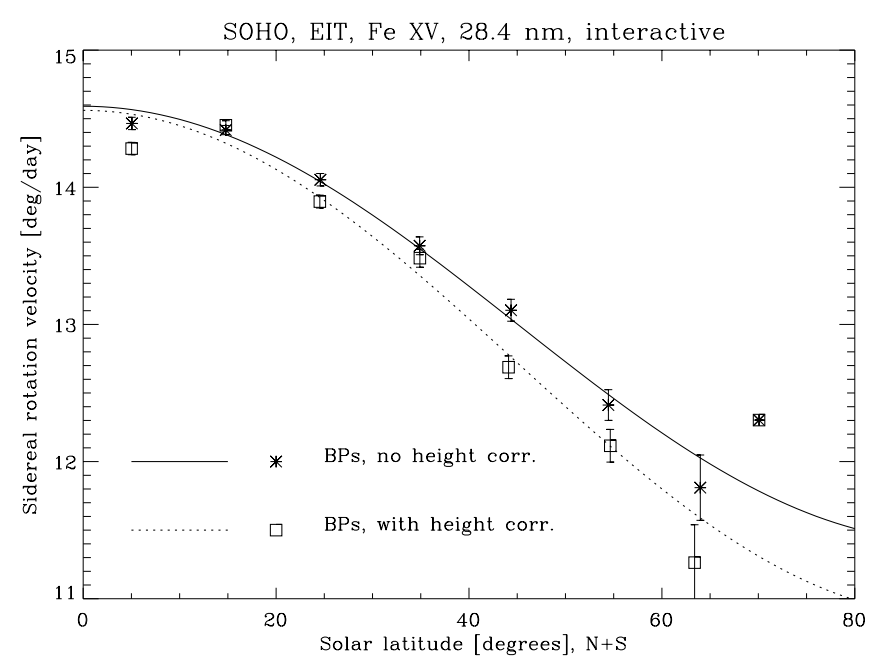

Fig. 3. The mean values of sidereal rotation velocities of coronal bright points (BPs) using the interactive method with and without the height correction, as indicated in the legend. The averaging is performed in 10-deg latitude bins and the error bars indicate the standard errors of the means in each bin (calculated as $M=\sigma / \sqrt{N}$, where $\sigma$ is the standard deviation and $N$ the number of data points in the bin). Both solar hemispheres are treated together and the lines represent least-squares fits $(C=0)$ to all filtered data points for each case. The parameters are given in the first lines in Tables 2 and 3, for the cases without and with height correction, respectively.

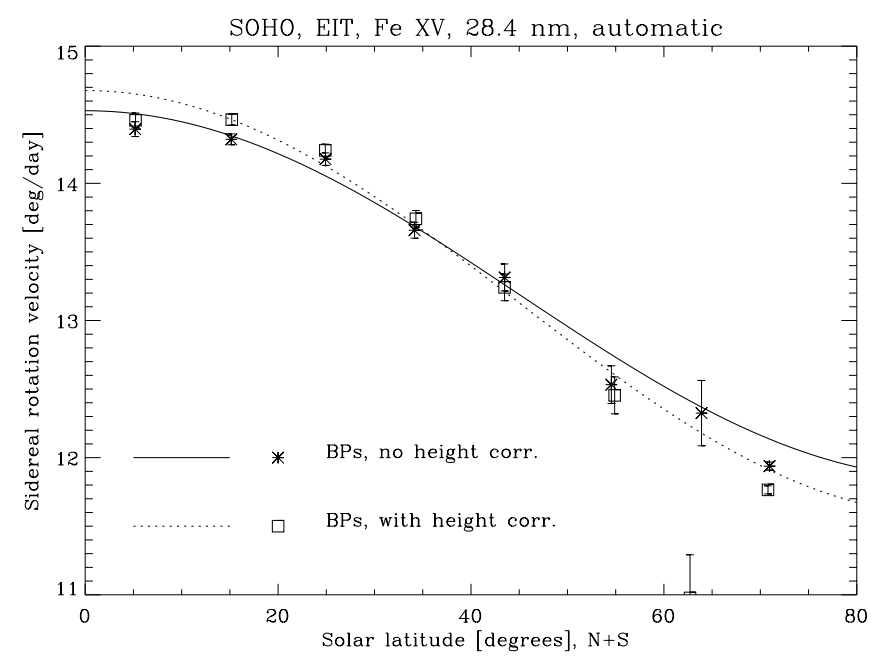

Fig. 4. As Fig. 3 for the automatic method (the case with $C=0$, Tables 4 and 5).

brightness temperature regions and magnetic features, as well as measuring Doppler shifts. Two or three parameters are calculated and in some cases only the equatorial rotation velocity, represented by the parameter $A$, is presented. When available, errors of the parameters are also given. Further details are listed as remarks below Table 6 .

Solar differential rotation curves obtained tracing coronal bright points are compared with some profiles obtained with other data in Figs. 5 and 6 for the interactive and automatic methods, respectively. In both cases data with and without the height correction are presented. 
Table 6. A comparison of differential rotation parameters $A, B$, and $C$ from Eq. (15) for coronal bright points (Tables 2-5) with other results. $\mathrm{R}$ - remark.

\begin{tabular}{|c|c|c|c|c|c|c|}
\hline Tracer/method & Time & $A \pm M_{A}$ & $-B \pm M_{B}$ & $-C \pm M_{C}$ & Source & $\mathrm{R}$ \\
\hline Coronal bright points & 1967 & $14.65 \pm 0.2$ & & & Dupree \& Henze (1972) & 1 \\
\hline Coronal bright points & 1973 & $14.6 \pm 0.3$ & & & Golub et al. (1974) & 2 \\
\hline Coronal bright points & 1998-1999 & $14.530 \pm 0.032$ & $2.68 \pm 0.13$ & & present work, Table 4 & 3 \\
\hline Coronal bright points & 1998-1999 & $14.677 \pm 0.033$ & $3.10 \pm 0.14$ & & present work, Table 5 & 4 \\
\hline Sunspots & 1921-1982 & $14.522 \pm 0.004$ & $2.84 \pm 0.04$ & & Howard et al. (1984) & 5 \\
\hline Sunspots & $1921-1982$ & $14.393 \pm 0.010$ & $2.95 \pm 0.09$ & & Howard et al. (1984) & 6 \\
\hline Sunspots & 1874-1976 & $14.551 \pm 0.006$ & $2.87 \pm 0.06$ & & Balthasar et al. (1986) & 7 \\
\hline Sunspots & 1853-1996 & $14.531 \pm 0.003$ & $2.75 \pm 0.05$ & & Pulkkinen \& Tuominen (1998) & 8 \\
\hline Sunspots & $1880-1976$ & $14.37 \pm 0.01$ & $2.59 \pm 0.16$ & & Brajša et al. (2002b) & 9 \\
\hline $\mathrm{H} \alpha$ filaments & 1919-1929 & 14.48 & 2.16 & & d'Azambuja \& d'Azambuja (1948) & 10 \\
\hline $\mathrm{H} \alpha$ filaments & $1972-1973$ & 14.45 & 1.43 & & Adams \& Tang (1977) & 11 \\
\hline $\mathrm{H} \alpha$ filaments & $1972-1987$ & $14.45 \pm 0.15$ & $0.11 \pm 0.90$ & $3.69 \pm 0.90$ & Brajša et al. (1991) & 12 \\
\hline Coronal bright points & 1998-1999 & $14.495 \pm 0.026$ & $1.89 \pm 0.06$ & $1.89 \pm 0.06$ & present work, Table 2 & 13 \\
\hline Coronal bright points & 1998-1999 & $14.454 \pm 0.027$ & $2.22 \pm 0.07$ & $2.22 \pm 0.07$ & present work, Table 3 & 14 \\
\hline LTRs & 1979-1991 & $14.25 \pm 0.06$ & $1.25 \pm 0.19$ & $1.25 \pm 0.19$ & Brajša et al. (2000a) & 15 \\
\hline LTRs & 1979-1991 & $14.16 \pm 0.06$ & $1.41 \pm 0.19$ & $1.41 \pm 0.19$ & Brajša et al. (2000a) & 16 \\
\hline Magnetic & $1967-1980$ & $14.307 \pm 0.005$ & $1.98 \pm 0.06$ & $2.15 \pm 0.11$ & Snodgrass (1983) & 17 \\
\hline Magnetic & $1975-1991$ & $14.42 \pm 0.02$ & $2.00 \pm 0.13$ & $2.09 \pm 0.15$ & Komm et al. (1993) & 18 \\
\hline Magnetic & 1997 & $14.00 \pm 0.54$ & $2.24 \pm 1.22$ & $1.78 \pm 0.79$ & Deng et al. (1999) & 19 \\
\hline Doppler & $1966-1968$ & 13.76 & 1.74 & 2.19 & Howard \& Harvey (1970) & 20 \\
\hline Doppler & $1967-1984$ & 14.05 & 1.49 & 2.61 & Snodgrass (1984) & 21 \\
\hline Doppler & 1981-1982 & $13.99 \pm 0.06$ & & & Küveler \& Wöhl (1983) & 22 \\
\hline Doppler & 1983-1986 & $13.92 \pm 0.12$ & & & Lustig \& Wöhl (1989) & 23 \\
\hline
\end{tabular}

1. OSO-4, Mg X spectroheliograms; 2. Skylab, soft X-rays; 3. SOHO-EIT, automatic method, no height correction;

4. SOHO-EIT, automatic method, with height correction; 5. Mt. Wilson, sunspots; 6. Mt. Wilson, sunspot groups;

7. Greenwich, sunspot groups; 8. Extended Greenwich, sunspots and sunspot groups;

9. Greenwich, only stable recurrent sunspot groups; 10. Meudon; 11. Big Bear, with height correction;

12. Big Bear and Kanzelhöhe, with height correction; 13. SOHO-EIT, interactive method, no height correction;

14. SOHO-EIT, interactive method, with height correction;

15. Metsähovi, microwave low brightness temperature regions, no height correction;

16. Metsähovi, microwave low brightness temperature regions, with height correction;

17. Mt. Wilson, photospheric magnetic fields; 18. Kitt Peak, photospheric magnetic fields;

19. Huairou/Beijing, photospheric high-latitude magnetic fields; 20. Mt. Wilson, photosphere, spectroscopic;

21. Mt. Wilson, photosphere, spectroscopic; 22. Locarno, photosphere, spectroscopic;

23. Capri, photosphere, spectroscopic.

From Table 6 and Fig. 5 we can see that coronal bright points traced with the interactive method rotate roughly as photospheric magnetic fields when a cross-correlation method is applied (Komm et al. 1993). On the other hand, coronal bright points traced with the automatic method rotate like sunspot groups (Balthasar et al. 1986) as can be seen in Table 6 and Fig. 6. The most probable explanation for this discrepancy is the difference in the latitudinal distribution of coronal bright points applying the two methods. Less than $2 / 3$ of all coronal bright points traced with the interactive method, but almost $3 / 4$ of them with the automatic method were identified in the latitude range from 10 to $40 \mathrm{deg}$ (as reported in a preliminary form by Brajša et al. 2003). So, the latitude distribution of coronal bright points traced with the automatic method has a more pronounced maximum at sunspot latitudes in comparison to the interactive method. For this reason the rotation velocity results obtained with the automatic method are similar to the ones determined by sunspot groups.

In this paper we have discussed the height correction in the determination of the solar differential rotation velocity. This is the most important source of systematic errors in the rotation velocity analysis for coronal tracers, especially when medium and high latitudes are considered. Other possible sources of errors include intrinsic proper motions due to changes in the magnetic field structure, evolution of the tracer, possible systematic inaccuracy of the positioning of the solar axis $\left(P\right.$ and $\left.B_{0}\right)$, and observer errors (Schröter 1985). In the present analysis the improved solar disc coordinates (solar radius and disc centre) obtained with the refined solar limb detection method (Auchère et al. 1998) were used for precise position measurements, and 


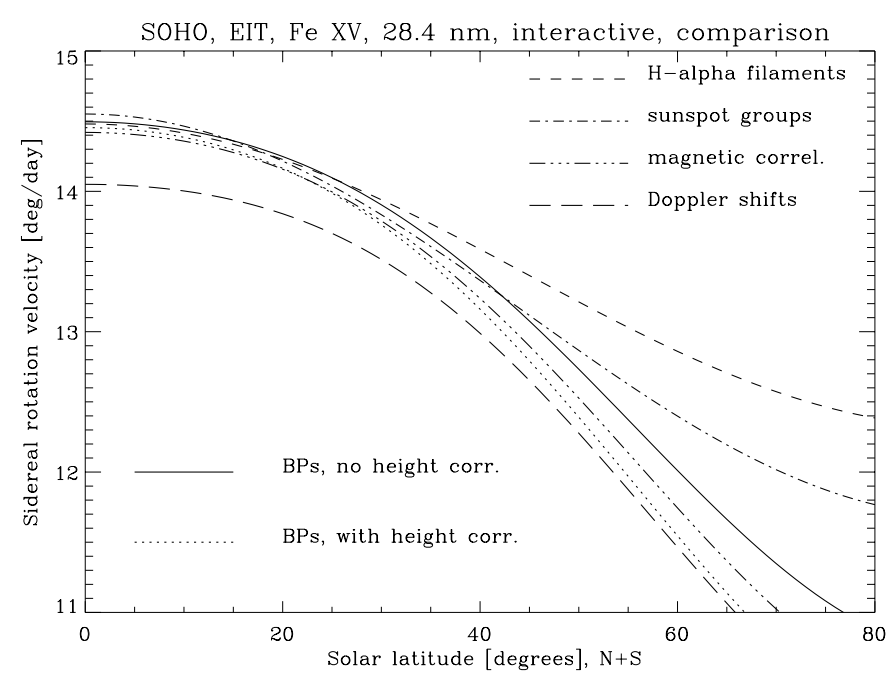

Fig. 5. Solar differential rotation curves for various tracers and methods. In the case of coronal bright points (BPs) the interactive method was used, and the results with and without the height correction are presented, as indicated in the legend (the case with $B=C$, Tables 2 and 3). The solar rotation parameters for $\mathrm{H} \alpha$ filaments, sunspot groups, magnetic correlations and Doppler shifts are specified with further details in Table 6 , in the lines 10, 7, 18, and 21, respectively.

the EIT plate scale was found to be constant across the field of view (Auchère et al. 2000). Structural and evolutionary changes of coronal bright points (Brajša et al. 2002a) may have some influence, but this is minimized in the present work taking the data set with shorter tracing times (Sect. 2). Possible observer errors due to different personnel who performed the tracings were discussed by Brajša et al. (2001b).

Finally, the "crosstalk" between the rotation parameters $B$ and $C$ may be a problem when the solar rotation velocity results are mutually compared, especially in the various phases of the solar activity cycle (Snodgrass 1984; Balthasar et al. 1986; Brajša et al. 1997). For this reason in the present work we have taken also the case with $B=C$ (Scherrer et al. 1980). However, the most important way to avoid crosstalk is to obtain the data from all latitudes and in this context coronal bright points, covering latitudes from the equator up to about $70 \mathrm{deg}$, have an obvious advantage.

We can conclude that coronal bright points are structures in the lower corona of the Sun having heights between $8000 \mathrm{~km}$ and $12000 \mathrm{~km}$ above the solar photosphere. They are magnetic tracers and the determined rotation velocity approximately corresponds to the velocity obtained for photospheric magnetic fields and sunspot groups by the interactive and automatic methods, respectively.

Acknowledgements. This work was performed with the support of the Alexander von Humboldt Foundation and is related to the SOHOEIT Proposal Brajsa_206: "An analysis of the solar rotation velocity by tracing coronal features" (http://umbra.nascom.nasa.gov/ eit/proposals/) submitted in March 1999 by R. Brajša, B. Vršnak, V. Ruždjak, D. Roša, H. Wöhl, and F. Clette. SOHO is a project of international cooperation between ESA and NASA. We would like to thank the EIT team for developing and operating the instrument, and research student T. J. Schuck for the help in preparing IDL programs

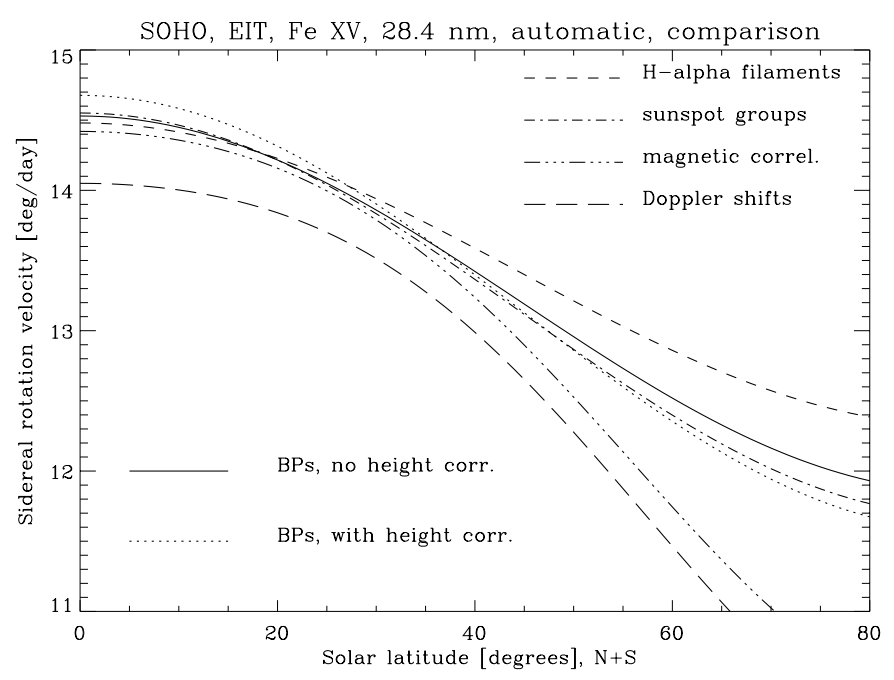

Fig. 6. As Fig. 5 for the automatic method (the case with $C=0$, Tables 4 and 5).

for the height correction. F. Clette and J.-F. Hochedez acknowledge the support from the Belgian OSTC and from Prodex.

\section{References}

Adams, W. M., \& Tang, F. 1977, Sol. Phys., 55, 499

Aschwanden, M. J., \& Bastian, T. S. 1994a, ApJ, 426, 425

Aschwanden, M. J., \& Bastian, T. S. 1994b, ApJ, 426, 434

Aschwanden, M. J., Lim, J., Gary, D. E., \& Klimchuk, J. A. 1995, ApJ, 454, 512

Auchère, F., Boulade, S., Koutchmy, S., et al. 1998, A\&A, 336, L57

Auchère, F., DeForest, C. E., \& Artzner, G. 2000, ApJ, 529, L115

Balthasar, H., \& Wöhl, H. 1983, Sol. Phys., 88, 71

Balthasar, H., Vázquez, M., \& Wöhl, H. 1986, A\&A, 155, 87

Brajša, R., Vršnak, B., Ruždjak, V., et al. 1991, Sol. Phys., 133, 195

Brajša, R., Ruždjak, V., Vršnak, B., et al. 1997, Sol. Phys., 171, 1

Brajša, R., Ruždjak, V., Vršnak, B., et al. 1999, Sol. Phys., 184, 281

Brajša, R., Ruždjak, V., Vršnak, B., et al. 2000a, Sol. Phys., 196, 279

Brajša, R., Wöhl, H., Kasabašić, M., et al. 2000b, Hvar Obs. Bull., 24, 153

Brajša, R., Wöhl, H., Vršnak, B., et al. 2001a, A\&A, 374, 309

Brajša, R., Wöhl, H., Schuck, T. J., et al. 2001b, Hvar Obs. Bull., 25, 13

Brajša, R., Wöhl, H., Vršnak, B., et al. 2002a, A\&A, 392, 329

Brajša, R., Wöhl, H., Vršnak, B., et al. 2002b, Sol. Phys., 206, 229

Brajša, R., Wöhl, H., Vršnak, B., et al. 2003, Hvar Obs. Bull., 27, 13

Brown, D. S., Parnell, C. E., DeLuca, E. E., Golub, L., \& McMullen, R. A. 2001, Sol. Phys., 201, 305

d'Azambuja, M., \& d'Azambuja, L. 1948, Ann. Obs. Paris-Meudon, VI, VII

Deng, Y., Wang, J., \& Harvey, J. 1999, Sol. Phys., 186, 13

Dupree, A. K., \& Henze, W., Jr. 1972, Sol. Phys., 27, 271

Fisk, L. A. 1996, J. Geophys. Res., 101, A7, 15547

Förster, M. 1979, Staatsexamensarbeit, Univ. of Göttingen

Gilman, P. A., \& DeLuca, E. E. 1986, in Cool Stars, Stellar Systems, and the Sun, Proc. IVth Cambridge Workshop, ed. M. Zeilik, \& D. M. Gibson (Berlin: Springer-Verlag), Lecture Notes in Phys., 254,163

Gissot, S. F., Hochedez, J.-F., Dibos, F., et al. 2003, Proc. ISCS Symp. 2003, ESA SP-535, 853

Gokhale, M. H. 1975, Sol. Phys., 41, 381 
Golub, L., Krieger, A. S., Silk, J. K., Timothy, A. F., \& Vaiana, G. S. $\quad$ Madjarska, M. S., Doyle, J. G., Teriaca, L., \& Banarjee, D. 2003, 1974, ApJ, 189, L93 A\&A, 398, 775

Golub, L., Rosner, R., Vaiana, G. S., \& Weiss, N. O. 1981, ApJ, 243, 309

Priest, E. R., Parnell, C. E., \& Martin, S. F. 1994, ApJ, 427, 459

Pulkkinen, P., \& Tuominen, I. 1998, A\&A, 332, 748

Hochedez, J.-F., Clette, F., Verwichte, E., Berghmans, D., \& Cugnon, P. 2001, IAU Symp., 203, 501

Howard, R., \& Harvey, J. 1970, Sol. Phys., 12, 23

Howard, R., Gilman, P. A., \& Gilman, P. I. 1984, ApJ, 283, 373

Roša, D., Vršnak, B., Božić, H., et al. 1998, Sol. Phys., 179, 237

Scherrer, P. H., Wilcox, J. M., \& Svalgaard, L. 1980, ApJ, 241, 811

Schröter, E. H. 1985, Sol. Phys., 100, 141

Snodgrass, H. B. 1983, ApJ, 270, 288

Komm, R. W., Howard, R. F., \& Harvey, J. W. 1993, Sol. Phys., 145, 1

Küveler, G., \& Wöhl, H. 1983, A\&A, 123, 29

Liu, S.-Y., \& Kundu, M. R. 1976, Sol. Phys., 46, 15

Snodgrass, H. B. 1984, Sol. Phys., 94, 13

Vršnak, B., Roša, D., Božić, H., et al. 1999, Sol. Phys., 185, 207

Vršnak, B., Brajša, R., Wöhl, H., et al. 2003, A\&A, 404, 1117

Longcope, D. W., Kankelborg, C. C., Nelson, J. L., \& Pevtsov, A. A. 2001, ApJ, 553, 429

Lustig, G., \& Wöhl, H. 1989, A\&A, 218, 299

Webb, D. F., Martin, S. F., Moses, D., \& Harvey, J. W. 1993, Sol. Phys., 144, 15

Wöhl, H., Brajša, R., Vršnak, B., et al. 2001, Hvar Obs. Bull., 25, 1 